\title{
Impact of Manure Storage Time and Temperature on Microbial Composition and Stable Fly (Diptera: Muscidae) Development
}

\author{
Janice L. Speshock ${ }^{1 *}$ (), Jeff A. Brady ${ }^{2,3}$, Jessica Eastman', \\ Travis Roach ${ }^{1}$, Samantha Hays ${ }^{4}$, David Kattes ${ }^{2}$ \\ ${ }^{1}$ Department of Biology, Tarleton State University, Stephenville, USA \\ ${ }^{2}$ Department of Wildlife, Sustainability \& Ecosystem Sciences, Tarleton State University, Stephenville, USA \\ ${ }^{3}$ Texas A\&M AgriLife Research \& Extension Center, Stephenville, USA \\ ${ }^{4}$ Department of Agriculture \& Environmental Science, Tarleton State University, Stephenville, USA \\ Email: *speshock@tarleton.edu
}

How to cite this paper: Speshock, J.L., Brady, J.A., Eastman, J., Roach, T., Hays, S. and Kattes, D. (2019) Impact of Manure Storage Time and Temperature on Microbial Composition and Stable Fly (Diptera: Muscidae) Development. Advances in Microbiology, 9, 248-265.

https://doi.org/10.4236/aim.2019.93018

Received: February 12, 2019

Accepted: March 24, 2019

Published: March 27, 2019

Copyright (๑) 2019 by author(s) and Scientific Research Publishing Inc. This work is licensed under the Creative Commons Attribution International License (CC BY 4.0).

http://creativecommons.org/licenses/by/4.0/

\begin{abstract}
Samples are often frozen for preservation until needed for use. It has been a common practice to store fresh dairy manure in the freezer until needed for fly development studies. However, conflicting data have suggested that freezer temperature and duration of manure may impact fly development studies, and it is likely due to the change in microbial comminutes due to the freezer conditions. In this study manure storage conditions were assessed to ascertain how temperatures impact stable fly, Stomoxys calcitrans L., survival to pupation and determine which bacterial populations impacted fly development using massively-parallel sequencing and $16 \mathrm{~S}$ metagenomic analysis. Stable fly survival to pupation was greater in manure that was stored warm $\left(27^{\circ} \mathrm{C}\right)$ or frozen $\left(-20^{\circ} \mathrm{C}\right.$ or $\left.-80^{\circ} \mathrm{C}\right)$ for 24 days as compared to fresh manure samples. Refrigeration $\left(4^{\circ} \mathrm{C}\right)$ of the manure for 24 days did not affect fly development and slightly decreased the pupal weights. Over 80 bacterial families were detected by sequencing allowing for a more thorough assessment of changes in bacterial populations. Only minor shifts were observed in bacterial family composition in the manure when refrigerated or frozen for 24 days, but significant population changes were observed when the manure was incubated for 24 days at $27^{\circ} \mathrm{C}$. Since it is the temperature and incubation time that yielded the greatest pupation rate, it is hypothesized that the manure microbial community impacts the growth and development of stable flies. This study has determined suggested freezer conditions for the best storage of manure samples to maintain bacterial diversity and retain the closest bacterial populations to freshly collected manure. Although untouched, aged (20 days) manure is best to use to assess fly development, it is not always feasible in la-
\end{abstract}


boratory experimentations. This study demonstrates the importance of preservation techniques on manure samples, which could also confer to storage of other biological specimens that contain resident microbes.

\section{Keywords}

Stomoxys calcitrans L., Manure Microbiota, 16S Metagenomics

\section{Introduction}

Stable flies, Stomoxys calcitrans L., are blood-sucking arthropods that can cause serious health issues for cattle and economic loss for cattle ranchers [1] [2]. They reduce productivity, lead to defense behaviors and bunching, and carry pathogens of cattle, which make stable flies a serious pest [2]. It is estimated that $139 \mathrm{~kg}$ of milk is lost per annum due to stable fly infestations resulting in an estimated economic loss of $\$ 360$ million each year for United States dairy cattle farmers [2]. It was originally thought that stable flies were only a pest problem for confined cattle, but more recent evidence indicates that stable flies can also impact pastured cattle [3]. The impact on pastured cattle is attributed to the use of hay bale feeders during the winter months, and more importantly, the manure accumulation around those feeders [4]. Due to insecticide costs and concern over insecticide-resistance, adequate pasture control of pest flies has been unattainable [1], which makes their development and control an important avenue of research.

Stable flies have shown a preference for aged manure (20 days) in which to oviposit, and larvae are rarely seen in fresh samples [5] [6]. There are many physicochemical features that may change between fresh and aged manure such as moisture content, temperature, $\mathrm{pH}$, and organic matter [5]. These parameter changes will have an effect on microbial composition, which has been demonstrated to impact stable fly larval development [1] [7]. Most studies examining bacterial communities in manure have relied on traditional plating methods, which can be unreliable since there are strict anaerobic bacteria, slow-growing bacteria, and bacteria that are easily out-competed by larger bacterial populations in the manure. Newer DNA sequencing technologies have enabled genomic analyses of bacterial population composition, which can include all organisms, regardless of growth parameters [8]. A study on horse manure used DNA pyrosequencing to assess changes in bacterial community composition and determined a decrease in overall species diversity with manure age and a shift from anaerobic to aerobic bacteria over time [6]. This shift in the microbial population could be contributing to the preferential environment that stable flies require in aged manure. It is, however, often challenging for researchers to store manure adequately, for the precise 18 - 20 days window for stable fly development studies. Therefore researchers have tried to utilize freezer conditions to collect large volumes and just extract samples when needed [9] [10] [11]. Here 
we demonstrated why freezing manure may complicate the consensus in fly development, and how studies performed on "ambient temperature" manure in various climates (warm or cold) may have drastic differences in results.

This study utilized a comparison of freshly collected dairy cattle manure to manure aged in a variety of temperatures at 1,7 , and $24 \mathrm{~d}$ post-collection, to determine the optimal storage conditions for stable fly development. The abundance of eggs developing to the pupal stage was compared to the bacterial composition, both using live culture-based assays and $16 \mathrm{~S}$ metagenomics, in order to determine factors that are important for the development of stable flies. The differences in storage conditions impact the bacteria microflora of the manure, which in turn will affect the development of stable flies. This data suggests that research on fly development and prevention needs to take storage into consideration and use ambient methods as much as possible to preserve the natural microbiome.

\section{Materials and Methods}

\subsection{Manure Collection}

Fresh bovine manure was collected from five random dairy cattle at the Southwest Regional Dairy Center in Stephenville, TX in October (average temperature $25^{\circ} \mathrm{C} / 14^{\circ} \mathrm{C}$ (high/low)). The manure was placed into a 19-L plastic bucket, thoroughly mixed and then separated into seventy-five 100 gram aliquots for the fly development studies and fifteen $10 \mathrm{~g}$ aliquots for the bacterial survey. Fifteen of the $100 \mathrm{~g}$ samples and three of the $10 \mathrm{~g}$ samples were placed into each of the following storage methods: outdoors $\left(27^{\circ} \mathrm{C}\right)$ covered with fly screen to prevent oviposition but allow for air movement, refrigeration $\left(4^{\circ} \mathrm{C}\right)$, freezer $\left(-20^{\circ} \mathrm{C}\right)$, and deep freeze $\left(-80^{\circ} \mathrm{C}\right)$ for a time period of 1,7 , or $24 \mathrm{~d}$. These storage conditions and time points were compared to manure that was processed the day of collection (fresh).

\subsection{Bacterial Population Survey}

On day 0 , using fresh manure, or days 1,7 , and $24 \mathrm{~d}$ old using manure stored at $27^{\circ} \mathrm{C}$ (covered), $27^{\circ} \mathrm{C}$ (uncovered), $4^{\circ} \mathrm{C},-20^{\circ} \mathrm{C}$, or $-80^{\circ} \mathrm{C}$, a 10 -g aliquot was removed and $5 \mathrm{~g}$ were used to make five $1 \%$ solutions in sterile phosphate-buffered saline (PBS). The treatment solutions were sonicated in a bath sonicator for 5 min. and were then vortexed for $1 \mathrm{~min}$. to distribute the bacterial populations evenly. Total prokaryotic cell counts were obtained by taking $100 \mu \mathrm{l}$ of each solution and adding it to $100 \mu \mathrm{l}$ of trypan blue and then manually counting using an inverted microscope and a Petroff-Hausser counting chamber (Hausser Scientific, Horsham, PA). Two replications from each manure solution were counted by two separate individuals for confirmation of accuracy. Following the counting, three replicates of each of the five solutions were plated onto eosin-methylene blue (EMB) agar for the determination of Escherichia coli concentrations in the manure samples. Lastly, Deoxyribonucleic acids (DNA) were 
extracted from $200 \mu \mathrm{l}$ of the manure solution as previously described [12] for massively parallel sequencing and $16 \mathrm{~S}$ metagenomic analysis of bacterial populations. DNA concentration was determined using a Qubit ${ }^{\circledR} 2.0$ fluorometer with a Qubit $^{\circledR}$ dsDNA HS Assay Kit according to manufacturer's instructions (Life Technologies, Carlsbad, CA). The DNA from each sample was normalized to a concentration of $5 \mathrm{ng} / \mu \mathrm{l}$ before subsequent analysis.

\subsection{Massively-Parallel Sequencing}

Bacterial $16 \mathrm{~S}$ amplicons were generated using primers $341 \mathrm{~F}$

(5'-CCTACGGGNGGCWGCAG-3') and 785R

(5'-GACTACHVGGGTATCTAATCC-3') that amplify the V3 and V4 regions [13] [14]. PCR amplification was accomplished through denaturation at 95 degrees Celsius for 3 minutes, followed by 35 cycles of 95 degrees Celsius for 10 seconds, 55 degrees Celsius for 30 seconds, and 72 degrees Celsius for 30 seconds. Barcodes were constructed with the same PCR protocol. Paired-end sequence data were generated on an Illumina MiSeq instrument using v3 600 cycle kits (Illumina, San Diego, CA), with dual 6 basepair index sequences attached to each amplicon during indexing PCR as described in the Illumina $16 \mathrm{~S}$ Metagenomic Sequencing Library Preparation protocol [15].

The raw sequencing reads were processed with a combination of QIIME [16], USEARCH [17] and FASTX-Toolkit [18] software packages, as well as a series of custom python scripts. Individual sequence tags were compared to the Greengenes 13.8 reference sequence database [19] using UCLUST [17] in order to pick referenced-based Operational Taxonomic Units (OTUs) at 97\% similarity. The sequencing dataset was rarified to an equal sequencing depth of 860 sequences per sample by randomly subsampling sequences from each sample without replacement in order to provide even measures of bacterial alpha- and beta-diversity and to have equal sequencing depth for the production of all figures, tables, and statistical analyses.

\subsection{Larvae Development}

Fly eggs were obtained from the Knipling-Bushland U. S. Livestock Insect Research Lab (Kerrville, TX). Manure was removed from each storage condition, or used fresh, and $100 \mathrm{~g}$ of the substrate was placed into 510-g plastic cups (Hefty ${ }^{\circledR}$, Pactiv Corporation, Lake Forest, IL) $(n=5)$. The manure was allowed to acclimate to room temperature and 50 stable fly eggs were added to each cup. The cups were placed into an incubator set at $27^{\circ} \mathrm{C}, 86 \% \mathrm{RH}$ at a photoperiod length of 14:10 L:D and the larvae were allowed to develop for eight days until eclosion. Following this incubation period, the pupae were enumerated and weighed.

\subsection{Statistical Analysis}

For the $16 \mathrm{~S}$ metagenomic analyses, unweighted UniFrac distance matrices were used in the calculation of diversity measures [20]. In order to determine if mi- 
crobial community composition was significantly different between samples, PERMANOVA and bootstrapped Mann-Whitney U tests were conducted using the QIIME package [21] [22]. Differences in microbial taxa between different storage times and temperatures were determined with NCSS statistical package (NCSS, Kaysville UT). Analysis of variance (ANOVA) tests were used to determine significant differences between the treatment groups for the fly and bacterial assays.

Stable fly percentage data was normalized using arcsine transformation before analysis. Percent stable fly development to the pupal stage and pupal weights were analyzed using Statistix 10 and a Least Significant Difference test (LSD) was used to spate multiple means where appropriate. Analysis of results was considered significant at $\mathrm{P}<0.05$ so specific $\mathrm{P}$ values are not presented.

\section{Results}

\subsection{Viable Cell Counts}

To determine the impact of various storage temperatures and times on the number of viable prokaryotic cells, cells were microscopically counted using trypan blue, which does not permeate the membranes of live cells, and compared to the fresh extraction of manure on day 0 . A significant decline in viable cell populations was evident following 20 days of storage using any of the storage conditions (Table 1). Growth at $27^{\circ} \mathrm{C}$ demonstrated a significant increase in viable cell numbers over the first week, which was predominantly prokaryotic when the storage vial was left uncovered (Table 1), but predominantly eukaryotic (fungi) when the storage vial was sealed (data not shown). For this reason, and the noticeable impact on fly development (Figure 4), the covered $27^{\circ} \mathrm{C}$ treatment group was omitted. Following refrigeration, the total viable prokaryotic cell counts decreased, but not significantly from control, except after 24 days of storage (Table 1). The freezer temperatures did not seem to impact viable cells much in the first week of storage, and did not seem to vary much from each other, although the $-20^{\circ} \mathrm{C}$ freezer did decline more rapidly, but they both did retain viable cells for the entire 24 day trial (Table 1). Due to the high prevalence of $E$. coli in manure samples, it was hypothesized that E. coli levels may impact the hatching of pupae and development of adult flies, thus $E$. coli viable cell

Table 1. Total Viable Prokaryotic Cell Count $\left(\log _{10}\right.$ cells $\left./ \mathrm{ml}\right) \pm$ SEM. Microscopic cell counts using trypan blue stain to differentiate between live and dead cells, with only viable cells included. ${ }^{*}$ Statistically significant $(\mathrm{P}<0.05)$ from control (fresh manure).

\begin{tabular}{ccccc}
\hline & Day 0 & Day 1 & Day 7 & Day 24 \\
\hline Fresh & $8.19(0.24)$ & & & \\
$27^{\circ} \mathrm{C}$ & & & \\
$4^{\circ} \mathrm{C}$ & & $9.13(0.95)^{*}$ & $7.14(0.07)^{*}$ & $6.99(0.01)^{*}$ \\
$-20^{\circ} \mathrm{C}$ & $8.16(0.76)$ & $7.06(0.26)^{*}$ & $6.38(0.07)^{*}$ \\
$-80^{\circ} \mathrm{C}$ & $8.01(0.50)$ & $7.47(0.31)$ & $6.31(0.02)^{*}$ \\
\hline
\end{tabular}


counts were also measured using EMB agar. At $27^{\circ} \mathrm{C}$ (uncovered) the E. coli levels significantly increased by day 7 , and although a decline was observed by day 24 , there was still a significantly higher level of viable $E$. coli cells in this treatment group as compared to control manure (Table 2). Refrigeration in manure appears to have little effect on the viability of $E$. coli as it remains unchanged throughout the entire time course (Table 2). A slight decline in E. coli populations was observed following deep freeze $\left(-80^{\circ} \mathrm{C}\right)$ and a significant decline was observed using a standard freezer $\left(-20^{\circ} \mathrm{C}\right)$, but neither appeared to be impacted by duration of freezing (Table 2 ).

\subsection{Sequencing Metrics}

Following quality filtering and joining of the paired-end reads, there were 538,24916 S DNA sequence tags for the 39 samples with an average length of 447 bp and an average Q score of 37.94. The lowest number of sequences per sample was 863. For all figures and statistical analyses of the sequencing data, the sequence dataset was randomly subsampled to an even depth of 863 sequences per sample.

\subsection{Bacterial Richness}

Shannon diversity metrics were used to analyze alpha diversity within the microbial populations comparing duration of storage (Figure 1(a)), temperature of storage (Figure 1(b)), or a combination of the parameters (Figure 1(c)). The rarefaction measure of species richness indicates that temperature (Figure 1(b)) has a greater impact than time (Figure $1(\mathrm{a})$ ) on the number of species present. A comparison of the collective temperatures at each of the time points demonstrates very little change in species number indicating that the duration of time may not be a critical parameter of manure storage and prokaryotic species richness (Figure 1(a)). Temperature did however appear to impact species richness, especially at 27 degrees Celsius (Figure 1(b)). The 27 degree temperature storage decreased the species richness (Figure $1(\mathrm{~b})$ ) with a significant impact observed after 24 days of storage (purple line; Figure 1(c)).

\subsection{Bacterial Sample Variability}

Beta diversity analysis was used to compare the amount of variability within the

Table 2. E. coli Cell Count $\left(\log _{10} \mathrm{cfu} / \mathrm{ml}\right) \pm$ SEM. E. coli counts from EMB plates. ${ }^{\star}$ Statistically significant $(\mathrm{P}<0.05)$ from control (fresh manure).

\begin{tabular}{ccccc}
\hline & Day 0 & Day 1 & Day 7 & Day 24 \\
\hline Fresh & $3.29(0.44)$ & & & \\
$27^{\circ} \mathrm{C}$ & & $3.65(0.38)$ & $5.12(0.91)^{*}$ & $4.73(0.32)^{*}$ \\
$4^{\circ} \mathrm{C}$ & & $3.59(0.70)$ & $3.56(0.17)$ & $3.68(0.15)$ \\
$-20^{\circ} \mathrm{C}$ & & $2.52(0.3 .6)^{*}$ & $2.34(0.43)^{*}$ & $2.30(0.28)^{*}$ \\
$-80^{\circ} \mathrm{C}$ & & $2.88(0.21)$ & $3.03(0.78)$ & $2.92(0.06)$ \\
\hline
\end{tabular}



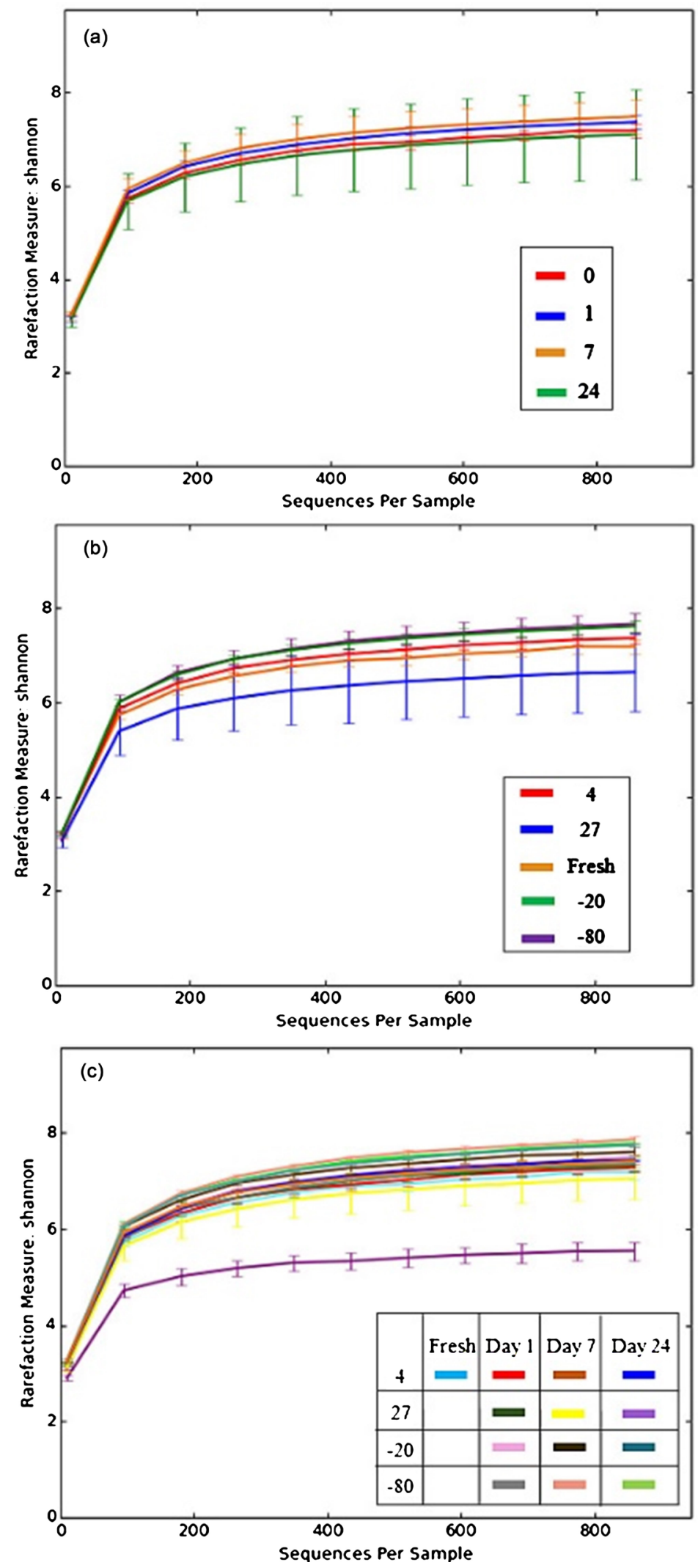

Figure 1. Alpha diversity plots obtained using Shannon diversity metrics. A comparison of duration (a), temperature (b), or combined duration and temperature parameters (c) was used to compare species richness within the samples. 
sample set and to examine the similarity between treatment groups. Principle coordinate analysis plots were used to demonstrate the variation present within the dataset to confirm consistent replicates and demonstrate effects of experimental parameters. For all storage temperatures and durations analyzed, there are no observable outliers from any of the three replicates, which is indicated by the close clustering of spheres of similar colors. With only one day of incubation (Figure 2(a)), there is still close clustering of the samples from various storage temperatures to the microbial DNA amplified from the freshly-obtained manure samples (red), suggesting that this short of an incubation time may not have a dramatic effect on bacterial species diversity. However, the frozen samples (blue -20 and orange -80 ) are a greater distance from the $27^{\circ} \mathrm{C}$ (purple) and refrigerated (green) samples, suggesting that even a short freeze may influence bacterial populations (Figure 2(a)). By day 7 of storage, most storage conditions have separated from the fresh manure samples (Figure 2(b)) indicating that the effects of temperature are starting to cause variability in the bacterial populations in the manure samples. The refrigerated samples still remain the most closely associated with the fresh manure after one week (Figure 2(b)). Following 24 days of storage, only the refrigerated samples remain in close proximity to the fresh manure (Figure $2(\mathrm{c})$ ), suggesting that refrigeration of manure will not dramatically change the prokaryotic cell population. The bacterial populations in the $27^{\circ} \mathrm{C}$ stored manure exhibited much more variation from the fresh manure samples following 24 days of storage, confirming the change in species richness observed in the alpha diversity plots (Figure 1(c) and Figure 2(c)). The frozen samples remained dissimilar from the fresh manure after 24 days of storage, but there appears to be little variation between freezing at -20 degrees Celsius or -80 degrees Celsius (Figure 2(c)).

\subsection{Bacterial Taxa Summary}

Although the primers used in this study are designed to selectively amplify bacterial $16 \mathrm{~S}$ sequences, a small number of $16 \mathrm{~S}$ sequence tags were generated from archaea. The archaea sequence tags were treated as contaminants for the purpose of this work, and are not discussed. Taxonomic changes of bacteria at the family level were examined between freshly obtained manure and manure stored at

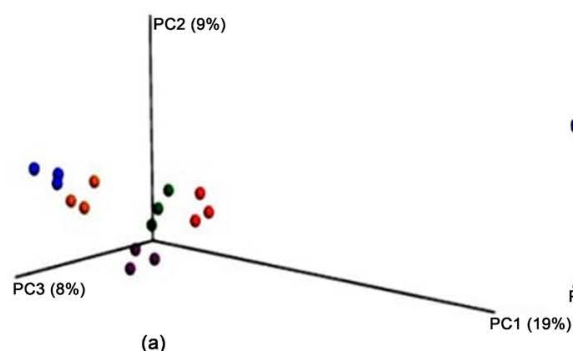

(a)

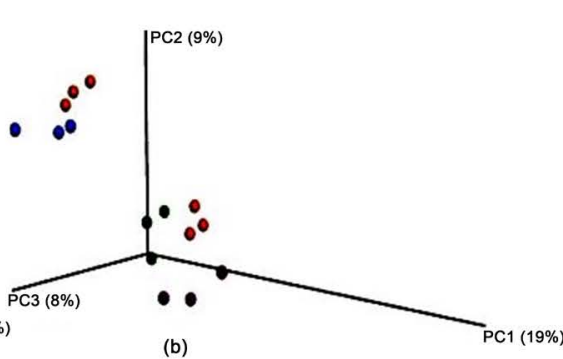

(b)

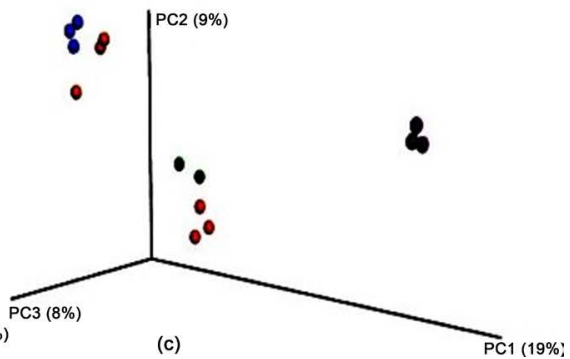

Figure 2. Unweighted UniFrac Beta Diversity Plots. Beta diversity plots comparing temperatures at 1 (a), 7 (b), and 24 (c) days of storage to freshly obtained manure (red spheres). The frozen manure is indicated by the blue $\left(-20^{\circ} \mathrm{C}\right)$ and orange $\left(-80^{\circ} \mathrm{C}\right)$ spheres. The $27^{\circ} \mathrm{C}$ stored manure $\left(27^{\circ} \mathrm{C}\right)$ is indicated by purple spheres. The refrigerated manure $\left(4^{\circ} \mathrm{C}\right)$ is indicated by green spheres. 
$-20^{\circ} \mathrm{C},-80^{\circ} \mathrm{C}, 4^{\circ} \mathrm{C}$, and $27^{\circ} \mathrm{C}$ (Figure S1). The only consistent change observed following 24 days in all storage conditions was in the Succinivibrionaceae family (Figure 3), which declined 9.8\%, 10.3\%, 5\%, and 8\%, respectively (Figure S1). Besides the Succinivibrionaceae family, there were no other changes between the fresh manure and the manure stored in the refrigerator for 24 days, and the Succinivibrionaceae were not quite statistically significant (Figure 3 ). More changes in population composition of various families were observed between the frozen manure $\left(-20^{\circ} \mathrm{C}\right.$ and $\left.-80^{\circ} \mathrm{C}\right)$ with little difference between the two temperatures, but the only large changes were observable increases in the Clostridiales order, although they were not statistically significant, and decreases in the family Bacteroidaceae, also not statistically significant (Figure 3). Decreases were also observed in percentage of Campylobacteraceae and Desulfovibrionaceae that were minor, but statistically significant. In the Clostridiales order, families "other" and "undefined" doubling in percent of composition, Clostridiaceae increasing from $1.4 \%$ in fresh manure to $3.3 \%\left(-20^{\circ} \mathrm{C}\right)$ or $4.0 \%\left(-80^{\circ} \mathrm{C}\right)$, Lachnospiraceae increasing from $7.1 \%$ in fresh manure to $8.8 \%\left(-20^{\circ} \mathrm{C}\right)$ or $10.6 \%\left(-80^{\circ} \mathrm{C}\right)$, and Ruminococcaceae increasing from $1.7 \%$ in fresh manure to $26.1 \%\left(-20^{\circ} \mathrm{C}\right)$ or

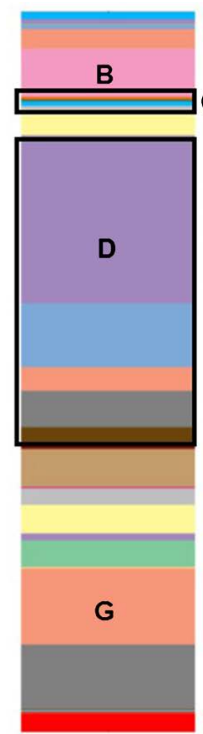

$-20^{\circ} \mathrm{C}$

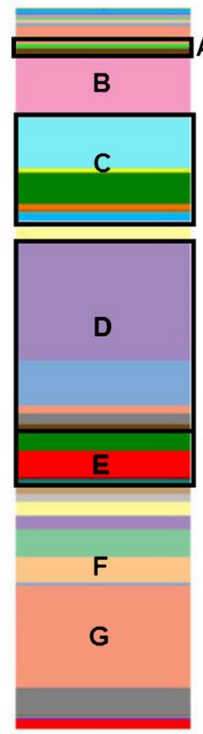

$27^{\circ} \mathrm{C}$

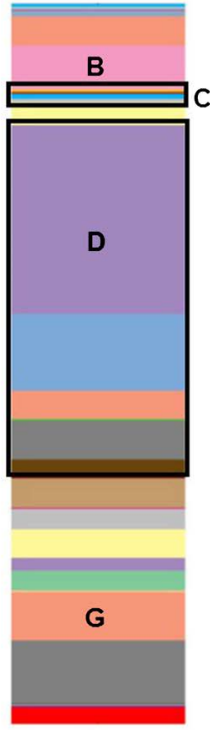

$-80^{\circ} \mathrm{C}$

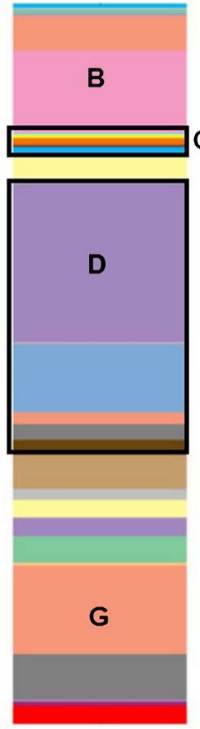

$4^{\circ} \mathrm{C}$

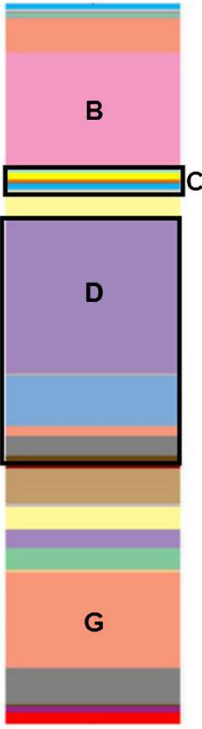

Fresh

Figure 3. Taxa summary bar graphs at the level of family. The percentage of prokaryote OTU representation of each family is compared at 24 days incubation between the various storage temperatures and a fresh manure control. The pink sections in the box labeled "A" depicts changes in Moraxellaceae (brown) and Xanthomonadaceae (green). The section labeled "B" depict Succinivibrionaceae. The box around sections labeled "C" includes the Campylobacterales (light blue), Desulfovibrionaceae (yellow), Comamonadaceae (green), Alcaligenaceae (orange), and Erysipelotrichaceae (dark blue) families. The box labeled " $\mathrm{D}$ " includes the families in Clostridiales order (families Ruminococcacae-purple, Lachnospiraceae (blue), Peptococcaceae-peach, undefined-grey, Christensenellaceae-green, other-brown). The box labeled "E" includes the Paraprevotellaceae (tan), Bacteroidales p-2534-18B5 (brown), Fibrobacteraceae (red), and Planococcaceae (green) families. The designations "F" and "G" are for the Porphyromonadaceae and Bacteroidaceae families, respectively. 
$23.3 \% 2\left(-80^{\circ} \mathrm{C}\right)$ (Figure 3; Figure S1). The most significant prokaryotic family shifts from the freshly obtained manure were observed in the manure stored at $27^{\circ} \mathrm{C}$ for 24 days (Figure 3 ). The percentage of OTU sequences from the family Paraprevotellaceae ( $\mathrm{P}=0.055$ ) decreased from $4.9 \%$ to $1.2 \%$ following a 24 day incubation at 27 degrees (Figure S1), and large increases were observed in the families Bacteroidales $\mathrm{P}-2534-18 \mathrm{~B} 5(\mathrm{P}=0.055)$, Porphyromonadaceae $(\mathrm{P}=$ 0.054), Fibrobacteraceae $(\mathrm{P}<0.05)$, Planococcaceae $(\mathrm{P}=0.051)$, Comamonadaceae $(\mathrm{P}<0.05)$, Campylobacteraceae $(\mathrm{P}=0.050)$, and Moraxellaceae $(\mathrm{P}<0.05)$ (Figure 3; Figure S1).

\subsection{Fly Development}

Fresh manure extracted from dairy cattle and then inoculated with stable fly eggs resulted in an average of 9.5 percent developing to the pupal stage (Figure 4(a)). The average weight from these control pupae was $0.036 \mathrm{~g}$ (Figure 4(b)). Manure stored at $-80^{\circ} \mathrm{C}$ for 24 days had an increase of about double the number pupae produced (20.1) and nearly double the average pupal size (0.068 g) (Figure 4(a) $\&$ Figure $4(\mathrm{~b}))$. There was little difference in pupation rate or average pupae size between manure frozen at $-20^{\circ} \mathrm{C}$ (data not shown) or $-80^{\circ} \mathrm{C}$, so the latter was used for all subsequent studies. When the manure was stored at $27^{\circ} \mathrm{C}$ uncovered for 24 days, there was a significant increase of over 3.5-fold for the number of eggs hatched (36.7 eggs) and a 2.7-fold significant increase for pupae weight (0.098 grams) (Figure 4(a) \& Figure 4(b)). Manure stored at $4^{\circ} \mathrm{C}$ appeared to have no impact on the number of eggs that hatched (Figure 4(a)), but the pupae that hatched were on average smaller (Figure $4(\mathrm{~b})$ ). The manure that was sealed and stored at $27^{\circ} \mathrm{C}$ had decreases in pupae number (Figure $4(\mathrm{a})$ ) and weight (Figure $4(\mathrm{~b})$ ), but these manure samples had an overgrowth of fungi, which
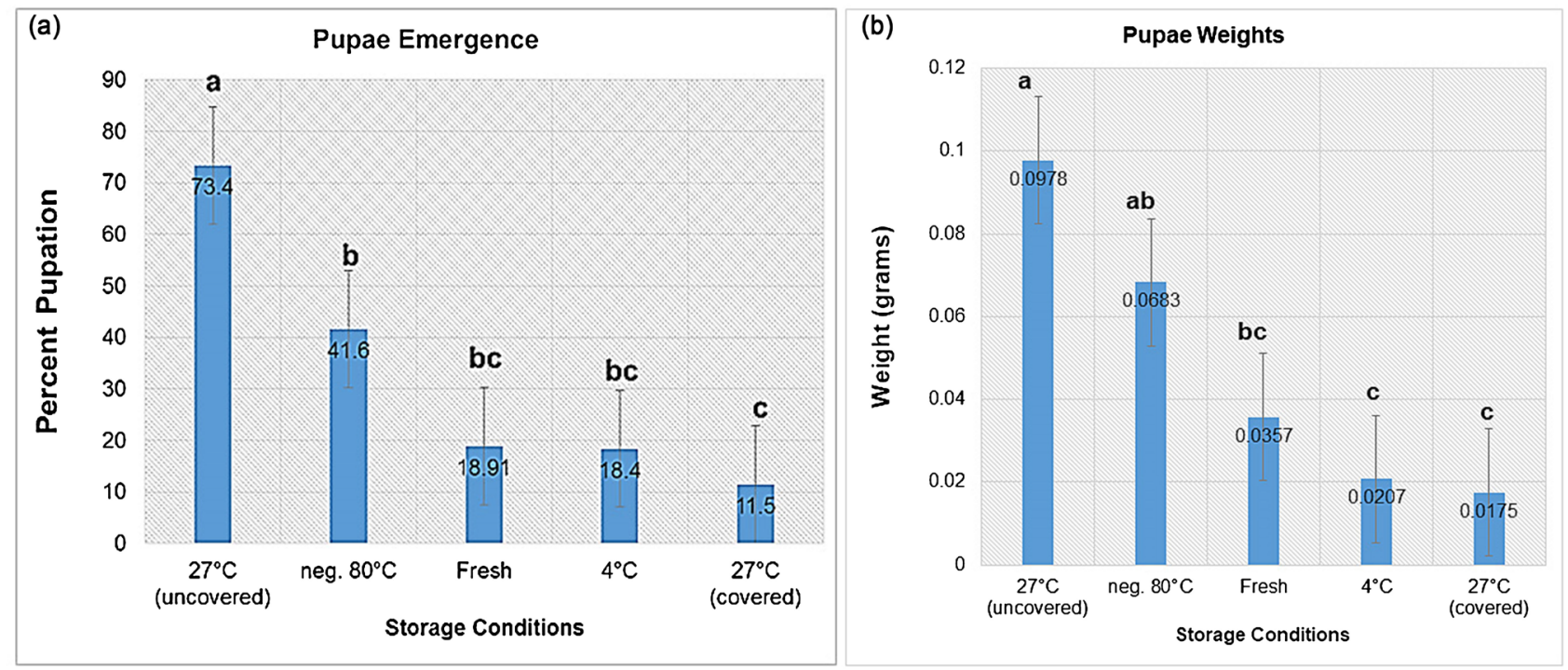

Figure 4. Pupae development. A comparison of pupae emergence from eggs (a) and the weight in grams of the pupae that emerged (b) was made following manure storage for 24 days at the various temperatures. Statistical significance $(\mathrm{P}<0.05)$ is denoted by different letters. 
likely impacted the development of stable flies.

\section{Discussion}

Due to the economic and medical importance of stable flies, much effort has been ongoing to attempt to better understand their breeding and development habits in order to reduce the fly populations around cattle and other livestock. As with many research objectives, there are many parameters that must be assessed to develop a full understanding of adequate methodology to obtain laboratory goals. The major question addressed in this research was does manure storage method and age impact bacterial growth, and in turn, stable fly larvae development? Often it is not feasible or practical to work with fresh manure samples, and thus researchers rely on storage of the manure until the protocols can proceed. Therefore we utilized common storage conditions, outdoors $\left(27^{\circ} \mathrm{C}\right)$, typical refrigerator setting $\left(4^{\circ} \mathrm{C}\right)$, a standard freezer $\left(-20^{\circ} \mathrm{C}\right)$, and a deep freeze $\left(-80^{\circ} \mathrm{C}\right)$, and compared manure samples stored in these conditions for 1 day, 1 week, and 24 days to determine how storage impacted fly larvae development and the bacterial populations in the manure.

The data from the 1 day storage was largely omitted from this manuscript, because it appeared to only be long-term storage that affected manure composition and quality. Although an overall viable cell number decrease was observed following refrigeration for 24 hours (Table 1), the overall percent composition of the microbes did not significantly change in this time period as compared with fresh manure (Figure 2). Conversely, an increase in total viable cell counts was measured following 24 hours at $27^{\circ} \mathrm{C}$ (Table 1), but again the overall species diversity was not significantly affected. There was an increase in species diversity and richness following 7 days of incubation, but since the data trended towards what was observed following 20 days of incubation, the data presented here focused mainly on the 24 day results.

Bacterial compositions have been implicated in the ability of stable fly larvae to develop, since sterilized media does not allow for any larval development [1] [6]. However, there are still many inconsistencies as to which bacteria are most important for fly development. Previous studies have relied on assessments of which bacteria grew best from manure, which most likely leads to the inconsistencies [1] [6] [23] [24]. Ascertaining community populations using standard agar plating techniques can be nearly impossible for bacteria since the growth parameters vary dramatically between organisms. Some bacteria require oxygen, while others do not. The optimal temperature of bacteria varies among species. Some species grow quickly (24 - 48 hours), while others take weeks to grow. Bacteria can out-compete other species for nutrients on agar plates. Most importantly however, is the fact that majority of bacteria in the gastrointestinal tract just do not grow in culture [25]. A recent advance in agar based assays was able to culture $88 \%$ of the human gut microbiota, which is far greater than any previous attempt, but is still lacking what could be important species [26]. This 
research began with examining $E$. coli levels since it was the largest constituent grown from the fresh manure cultures obtained, with $32 \%$ of all colonies on the agar plate representing E. coli (data not shown). However, further genomic analyses indicated that the prevalence of $E$. coli using sequence-based determination was less than $0.0002 \%$ (data not shown) of the bacterial population of the manure, confirming that standard plate techniques can be misleading. Metagenomic analysis provides a more thorough examination of the gut microbiota, and was used here to determine how changes in bacterial populations can impact stable fly larvae development.

The average pupation rate using fresh manure was $20 \%$, and this rate doubled in frozen manure, and was greater than $70 \%$ with manure stored at $27^{\circ} \mathrm{C}$ for 24 days (Figure 4(a)). Pupae size followed a similar trend with $27^{\circ} \mathrm{C}$ incubation for 24 days resulting in the heaviest pupae, which were slightly heavier than from manure that was frozen for 24 days, and both of these stored samples produced heavier pupae than fresh manure (Figure 4(b)). Refrigeration of the manure had little effect on pupation rate compared to fresh, but did result in pupae of smaller size. After determination of how temperatures and durations impact stable fly development, the next question addressed was what changed in the $27^{\circ} \mathrm{C}$ or frozen manure to allow for increased pupation rate and pupae size?

The number of viable prokaryotic cells increased during the first week of incubation at $27^{\circ} \mathrm{C}$, but the cells eventually died off during the 24 day incubation, resulting in a significant decrease in total viable cells from the fresh sample (Table 1). The number of viable cells declined over time in both freezer temperatures resulting in a significant decrease in viable prokaryotic cells following 24 days of storage similar to the amount observed following 24 days of incubation at $27^{\circ} \mathrm{C}$ (Table 1 ). If this cell death trend is a necessary factor in stable fly development, it may indicate the need for secondary metabolites produced and released during the stationary and death phases of bacterial growth as opposed to the bacteria themselves. However, a similar trend of cell death was also observed with manure refrigerated for 24 days (Table 1), which resulted in no increase in pupation rate, and a decrease in average pupae weight, suggesting that perhaps specific species of bacteria are indeed needed for stable fly growth. Escherichia coli was selected for further study since $E$. coli growth far outnumbered other bacterial species when fresh manure was plated on Mannitol Salt Agar and Eosin Methylene Blue agar and incubated overnight at $37^{\circ} \mathrm{C}$, resulting in $32 \%$ of all observed colonies on the two plates (data not shown). The E. coli populations increased over the first week of incubation at $27^{\circ} \mathrm{C}$, but declined a little after 24 days (Table 2). However, even after the slight decline at the 24 day time point, the $E$. coli levels were still significantly higher than fresh manure $E$. coli levels, suggesting that this organism may be contributing to the increase in pupae observed after 24 days of incubation at $27^{\circ} \mathrm{C}$. After determination of the E. coli levels following 24 days of refrigeration or freezing, this hypothesis was refuted since refrigerated manure has greater levels of $E$. coli than fresh manure, but re- 
sulted in comparable fly pupation, and frozen manure had significantly less $E$. coli, but had greater pupation than fresh manure (Table 2; Figure 4).

Previous studies have attempted to elucidate bacteria that are important for stable fly development, but they too relied on culture methods, which can be misleading [1] [6]. Both of these previous studies cultured bacteria from manure in aerobic conditions, but analysis of the bacterial phyla in ruminant animals indicates that a majority of the fecal microbiome consists of species from the phyla Bacteroidetes, Firmicutes, and Proteobacteria, all three of which contain mostly anaerobic species [27]. All families that had significant changes resulting from the various storage conditions tested here belonged to one of these three phyla, indicating that aerobic plating methods may not have allowed for easy depiction of the importance of these bacterial families to stable fly development. It is hypothesized that changes in population diversity among the phyla Bacteroidetes, Firmicutes, and Proteobacteria had the greatest contribution to increased stable fly pupation.

An approximate doubling of average eggs hatched and pupae size was observed when the manure was stored for 24 days at $-80^{\circ} \mathrm{C}$ compared to fresh manure, although due to sample variation it was not a significant increase (Figure 4). The frozen samples $\left(-20^{\circ} \mathrm{C}\right.$ or $\left.-80^{\circ} \mathrm{C}\right)$ had an increase in prokaryotic species richness (Figure 2(b)) and species variation (Figure 2(c)) over the fresh manure control (Figure 1(b)), suggesting a good preservation of the microbial population. The manure stored at $27^{\circ} \mathrm{C}$ for 24 days allowed for a nearly 4 -fold increase in hatch number and 2.7-fold increase in pupae size, both of which were statistically significant, over fresh manure pupation rates and growth (Figure 4). However, this manure actually demonstrated a dramatic decrease in bacterial species diversity at 24 days with even a slight, but noticeable decline at 7 days incubation (Figure 1(c)). The beta diversity analysis also indicated an increase in species variation, suggesting that the incubation at this temperature promoted the growth of certain bacterial species, while inhibiting the growth (or killing off) others (Figure 2 and Figure 3). All observed changes in bacterial families originated in the phyla Bacteroidetes, Firmicutes, and Proteobacteria, but no discernable trend can be established to suggest that one of these phyla contributed greatly to the abundance of stable flies.

For the manure incubated at $-20^{\circ} \mathrm{C}$ or $-80^{\circ} \mathrm{C}$, the only increase was observed in families of the Clostridiales order; Firmicutes phylum (families Clostridiaceae, Lachnospiraceae, and Ruminococcaceae) (Figure 3; Figure S1). Species of the Clostridiales order are anaerobic bacteria found in the gastrointestinal tracts of most mammals that form endospores, which are dormant, protective structures of bacteria, and this may have contributed to their survivability in the freezer temperatures. Clostridiaceae are rapidly able to assimilate glucose to glycogen for energy, or dissimilate it into many metabolites (acetate, butyrate, lactate, ethanol, dihydrogen, and carbon dioxide) [28]. Ruminococcaceae ferment cellulose and produce ethanol as a waste product [29]. Stable flies have been shown to 
be attracted to a combination of acetic acid and ethanol, thus this glucose dissimilation by Clostridiaceae may be producing a chemoattractant for the flies [30]. Lachnospiraceae have been implicated in the ability to stimulate host epithelial cell growth due to the production of butyric acid [31].

The manure stored at $27^{\circ} \mathrm{C}$ for 24 days had many observable changes in bacterial families, all from the phyla Bacteroidetes, Firmicutes, and Proteobacteria. No information was found regarding the unclassified Bacteroidetes family Bacteroidales p2534-18B5, but the other family with increases in species composition, Porphyromonadaceae and a closely-related family, Fibrobacteraceae, that used to be linked to the phylum, are both beneficial bacteria that aid in digestion of complex carbohydrates [32] [33]. Porphyromonadaceae digest fiber and other carbohydrates into short-chain fatty acids, such as butyrate, which is more digestible and has been shown to aid in healthy digestion and colon health [33]. Fibrobacteraceae are highly fibrolytic and cellulytic bacteria that aid in digestion and produce storage carbohydrates glycogen and cellodextrins [32]. Although the Firmicutes displayed large variation in manure stored at $-20^{\circ} \mathrm{C}$ and $-80^{\circ} \mathrm{C}$, when stored at $27^{\circ} \mathrm{C}$ only one family had any change, which were the Planococcaceae which have been found in the rumen by other analyses [34], but whose function in digestion remains unknown. The remaining family diversity changes were observed in the phylum Proteobacteria, the largest and most diverse phylum of the bacteria. Significant increases were observed in the families Comamonadaceae, denitrifying bacteria, the Campylobacteraceae, chemoorganotrophs that reduce nitrate, and Moraxellaceae, which have been implicated in infectious bovine keratoconjunctivitis (IBK), but have not been reported in the rumen or fecal material [35] [36] [37].

Although a specific pattern was not formed to identify a particular family or species of bacteria implicated in the growth and development of stable flies, this study was able to assess the impact of fastidious, anaerobic bacterial families that are likely impacting stable fly pupation. Previous studies have relied on laboratory growth of bacteria in aerobic conditions, which limits some of the most prevalent species of gut microorganisms from assessment. Increases in bacteria that aid ruminants in digestion of complex carbohydrates resulting in the production of fermentation products of ethanol or butyrate (or butyric acid) or storage carbohydrates such as glycogen and cellodextrins, may be providing a food source for the flies. Alternatively, a need for a more bioavailable form of nitrogen might be a requirement for stable fly pupation, and the Proteobacteria may be providing a nitrogen source through their nitrogen catabolism pathways. Growth at $27^{\circ} \mathrm{C}$, more accurately mimics a likely scenario of what is occurring in the cattle fields, thereby further studies in bacterial families observed to increase here may elucidate the bacterial composition, and catabolism products, required for stable fly growth and development. Regardless, it is imperative that researchers in the fields of manure microbiome research and fly development take into account the temperature and duration of storage of the manure and know that 
these parameters could drastically impact the results obtained. This knowledge is not only important for manure studies, but for any culture preservation that involves preserving a microbiome.

\section{Acknowledgements}

We would like to thank Dr. Kim Lohmeyer and Mr. Matt Waldon from the Agricultural Research Services of the United States Department of Agriculture in Kerrville, Texas for the stable fly eggs. We would also like to thank the Tarleton State University Office of Student Research and Creative Activities for funding the project.

\section{Conflicts of Interest}

The authors declare no conflicts of interest regarding the publication of this paper.

\section{References}

[1] Romero, A., Broce, A. and Zurek, L. (2006) Role of Bacteria in the Ovipostion Behavior and Larval Development of Stable Flies. Medical and Veterinary Entomology, 20, 115-121. https://doi.org/10.1111/j.1365-2915.2006.00602.x

[2] Taylor, D.B., Moon, R.D. and Mark, D.R. (2012) Economic Impact of Stable Flies (Diptera: Muscidae) on Dairy and Beef Cattle Production. Journal of Medical Entomology, 49, 198-209. https://doi.org/10.1603/ME10050

[3] Campbell, J.B., Skoda, S.R., Berkebile, D.R., Boxler, D.J., Thomas, G.D., Adams, D.C. and Davis, R. (2001) Effects of Stable Flies (Diptera: Muscidae) on Weight Gains of Grazing Yearling Cattle. Journal of Economic Entomology, 94, 780-783. https://doi.org/10.1603/0022-0493-94.3.780

[4] Talley, J.A., Broce, B. and Zurek, L. (2009) Characterization of Stable Fly (Diptera: Muscidae) Larval Development Habitat at Round Hay Bale Feeding Sites. Journal of Medical Entomology, 46, 1310-1319. https://doi.org/10.1603/033.046.0609

[5] Broce, A.B. and Haas, M. (1999) Relation of Cattle Manure Age to Colonization by Stable Fly and House Fly (Diptera: Muscidae). Journal of the Kansas Entomological Society, 72, 60-72.

[6] Zurek, L. and Albuquerque, T. (2008) Microbial Ecology of Stables Flies: Effect of Bacterial Community of Aging Horse Manure on Stable Fly Fitness. 17 th Annual K-State Research Forum, Manhattan, KS, 8 March 2012.

[7] Lysyk, T.J., Kalischuk-Tymensen, L., Selinger, L.B., Lancaster, R.C., Wever, L. and Cheng, K.J. (1999) Rearing Stable Fly Larvae (Diptera: Muscidae) on an Egg Yolk Medium. Journal of Medical Entomology, 36, 382-388.

https://doi.org/10.1093/jmedent/36.3.382

[8] Caporaso, J.G., Lauber, C.L., Walters, W.A., et al. (2012) Ultra-High-Throughput Microbial Community Analysis on the Illumina HiSeq and MiSeq Platforms. The ISME Journal, 6, 1621-1624. https://doi.org/10.1038/ismej.2012.8

[9] Myers, H.M., Tomberlin, J.K., Lambert, B.D. and Kattes, D. (2008) Development of Black Soldier Fly (Diptera: Stratiomyidae) Larvae Fed Dairy Manure. Environmental Entomology, 37, 11-15. https://doi.org/10.1093/ee/37.1.11

[10] Moon, R.D., Hinton, J.L., O’Rourke, S.D. and Schmidt, D.R. (2001) Nutritional 
Value of Fresh and Composted Poultry Manure for House Fly (Diptera: Muscidae) Larvae. Journal of Economic Entomology, 94, 1308-1317. https://doi.org/10.1603/0022-0493-94.5.1308

[11] Geden, C.J. (1999) Host Location by House Fly (Diptera: Muscidae) Parasitoids in Poultry Manure at Different Moisture Levels and Host Densities. Environmental Entomology, 28, 755-760. https://doi.org/10.1093/ee/28.4.755

[12] Brady, J.A., Faske, J.B., Castañeda-Gill, J.B., King, J.L. and Mitchell, F.L. (2011) High-Throughput DNA Isolation Method for Detection of Xylella fastidiosa in Plant and Insect Samples. Journal of Microbiological Methods, 86, 310-312. https://doi.org/10.1016/j.mimet.2011.06.007

[13] Herlemann, D.P.R., Labrenz, M., Jurgens, K., Bertilsson, S., Waniek, J.J. and Andersson, A.F. (2011) Transitions in Bacterial Communities along the $2000 \mathrm{~km}$ Salinity Gradient of the Baltic Sea. The ISME Journal, 5, 1571-1579. https://doi.org/10.1038/ismej.2011.41

[14] Klindworth, A., Pruesse, E., Schweer, T., Peplies, J., Quast, C., Horn, M. and Glöckner, F.O. (2012) Evaluation of General 16S Ribosomal RNA Gene PCR Primers for Classical and Next-Generation Sequencing-Based Diversity Studies. Nucleic Acids Research, 41, e1. https://doi.org/10.1093/nar/gks808

[15] Illumina (2013) 16S Metagenomic Sequencing Library Preparation.

[16] Caporaso, J.G., Kuczynski, J., Stombaugh, J., et al. (2010) QIIME Allows Analysis of High-Throughput Community Sequencing Data. Nature Methods, 7, 335-336. https://doi.org/10.1038/nmeth.f.303

[17] Edgar, R.C. (2010) Search and Clustering Orders of Magnitude Faster than BLAST. Bioinformatics, 26, 2460-2461. https://doi.org/10.1093/bioinformatics/btq461

[18] Hannon. FASTX Toolkit. http://hannonlab.cshl.edu/fastx_toolkit/

[19] DeSantis, T.Z., Hugenholtz, P., Larsen, N., et al. (2006) Greengenes, a Chimera-Checked 16S rRNA Gene Database and Workbench Compatible with ARB. Applied and Environmental Microbiology, 72, 5069-5072. https://doi.org/10.1128/AEM.03006-05

[20] Lozupone, C. and Knight, R. (2005) UniFrac: A New Phylogenetic Method for Comparing Microbial Communities. Applied and Environmental Microbiology, 71, 8228-8235. https://doi.org/10.1128/AEM.71.12.8228-8235.2005

[21] Anderson, M.J. (2001) A New Method for Non-Parametric Multivariate Analysis of Variance. Austral Ecology, 26, 32-46.

[22] Anderson, M.J., Crist, T.O., Chase, J.M., et al. (2011) Navigating the Multiple Meanings of Beta Diversity: A Roadmap for the Practicing Ecologist. Ecology Letters, 14, 19-28. https://doi.org/10.1111/j.1461-0248.2010.01552.x

[23] Schmidtmann, E.T. and Martin, P.A.W. (1992) Relationship between Selected Bacteria and the Growth of Immature House Flies, Musca domestica, in an Axenic Test System. Journal of Medical Entomology, 29, 232-235. https://doi.org/10.1093/jmedent/29.2.232

[24] Zurek, L., Schal, C. and Watson, D.W. (2000) Diversity and Contribution of the Intestinal Bacterial Community to the Development of Musca domestica (Diptera: Muscidae) Larvae. Journal of Medical Entomology, 37, 924-928. https://doi.org/10.1603/0022-2585-37.6.924

[25] Wade, W. (2002) Unculturable Bacteria-The Uncharacterized Organisms That Cause Oral Infections. Journal of the Royal Society of Medicine, 95, 81-83.

[26] Rettedal, E.A., Gumpert, H. and Sommer, M.O.A. (2014) Cultivation-Based Mul- 
tiplex Phenotyping of Human Gut Microbiota Allows Targeted Recovery of Previously Uncultured Bacteria. Nature Communications, 5, Article No. 4714. https://doi.org/10.1038/ncomms5714

[27] Jami, E. and Mizrahi, I. (2012) Composition and Similarity of Bovine Rumen Microbiota across Individual Animals. PLoS ONE, 7, e33306.

https://doi.org/10.1371/journal.pone.0033306

[28] Wust, P.K., Horn, M.A. and Drake, H.L. (2011) Clostridiaceae and Enterobacteriaceae as Active Fermentaers in Earthworm Gut Content. The ISME Journal, 5, 92-106. https://doi.org/10.1038/ismej.2010.99

[29] Tian, Z., Cabrol, L., Ruiz-Filippi, G. and Pullammanappallil, P. (2014) Microbial Ecology in Anaerobic Digestion at Agitated and Non-Agitated Conditions. PLoS ONE, 9, e109769. https://doi.org/10.1371/journal.pone.0109769

[30] Ladnolt, P.J., Cha, D.H. and Zack, R.S. (2015) Synergistic Trap Response of the False Stable Fly and Little House Fly (Diptera: Muscidae) to Acetic Acid and Ethanol, Two Principle Sugar Fermentation Volatiles. Environmental Entomology, 44, 1441-1448. https://doi.org/10.1093/ee/nvv119

[31] Meehan, C.J. and Beiko, R.G. (2014) A Phylogenomic View of Ecological Specialization in the Lachnospiraceae, a Family of Digestive Tract-Associated Bacteria. Genome Biology and Evolution, 6, 703-713. https://doi.org/10.1093/gbe/evu050

[32] Qi, M., Nelson, K.E., Daugherty, S.C., Nelson, W.C., Hance, I.R., Morrison, M. and Forsberg, C.W. (2005) Novel Molecular Features of the Fibrolytic Intestinal Bacterium Fibrobacter intestinalis Not Shared with Fibrobacter succinogenes as Determined by Suppressive Subtractive Hybridization. Journal of Bacteriology, 187, 3739-3751. https://doi.org/10.1128/JB.187.11.3739-3751.2005

[33] Zackular, J.P., Baxter, N.T., Iverson, K.D., Sadler, W.D., Petrosino, J.F., Chen, G.Y. and Schloss, P.D. (2013) The Gut Microbiome Modulates Colon Tumorigenesis. mBio, 4, e00692-13. https://doi.org/10.1128/mBio.00692-13

[34] Kim, M., Morrison, M. and Yu, Z. (2011) Status of the Phylogenetic Diversity Census of Ruminal Microbiomes. FEMS Microbiology Ecology, 76, 49-63. https://doi.org/10.1111/j.1574-6941.2010.01029.x

[35] Vandamme, P. and De Ley, J. (1991) Proposal for a New Family, Campylobacteraceae. International Journal of Systematic and Evolutionary Microbiology, 41, 451-455. https://doi.org/10.1099/00207713-41-3-451

[36] Khan, S.T., Horiba, Y., Yamamoto, M. and Hiraishi, A. (2002) Members of the Family Comamonadaceae as Primary Poly(3-Hydroxybutyrate-Co-3-Hydroxyvalerate)Degrading Denitrifiers in Activated Sludge as Revealed by a Polyphasic Approach. Journal of Applied \& Environmental Microbiology, 68, 3206-3214. https://doi.org/10.1128/AEM.68.7.3206-3214.2002

[37] Angelos, J.A. and Ball, J.M. (2007) Differentiation of Moraxella bovoculi sp. nov. from Other Coccoid Moraxellae by the Use of Polymerase Chain Reaction and Restriction Endonuclease Analysis of Amplified DNA. Journal of Veterinary Diagnostic Investigation, 19, 532-534. https://doi.org/10.1177/104063870701900511 


\section{Supplement}

\begin{tabular}{|c|c|c|c|c|c|}
\hline Taxon & -20 & 27 & -80 & 4 & Fresh \\
\hline None;Other;Other;Other;Other & $2.5 \%$ & $1.1 \%$ & $2.3 \%$ & $2.6 \%$ & $1.6 \%$ \\
\hline k_Archaea;p_Euryarchaeota;c_Methanobacteria;o__Methanobacteriales;f__Methanobacteriaceae & $0.1 \%$ & $0.1 \%$ & $0.0 \%$ & $0.0 \%$ & $0.0 \%$ \\
\hline k_Bacteria;Other;Other;Other;Other & $0.2 \%$ & $0.4 \%$ & $0.2 \%$ & $0.5 \%$ & $0.8 \%$ \\
\hline 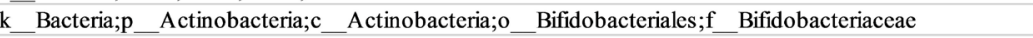 & $0.1 \%$ & $0.1 \%$ & $0.1 \%$ & $0.0 \%$ & $0.0 \%$ \\
\hline k_ Bacteria;p_Actinobacteria;c_Coriobacteriia;o__Coriobacteriales; $;$ Coriobacteriaceae & $0.1 \%$ & $0.0 \%$ & $0.1 \%$ & $0.0 \%$ & $0.0 \%$ \\
\hline k__Bacteria;p__Bacteroidetes;c__Bacteroidia;o__Bacteroidales;Other & $0.1 \%$ & $0.1 \%$ & $0.1 \%$ & $0.1 \%$ & $0.3 \%$ \\
\hline $\mathrm{k}$ __Bacteria;p__Bacteroidetes;c__Bacteroidia;o__Bacteroidales;f & $9.0 \%$ & $3.8 \%$ & $8.8 \%$ & $6.4 \%$ & $5.0 \%$ \\
\hline k_Bacteria;p_Bacteroidetes;c__Bacteroidia;o__Bacteroidales;f__BS11 & $0.1 \%$ & $0.1 \%$ & $0.0 \%$ & $0.0 \%$ & $0.0 \%$ \\
\hline k___Bacteria;p__Bacteroidetes;c__Bacteroidia;o__Bacteroidales;f__Bacteroidaceae & $10.5 \%$ & $14.2 \%$ & $6.7 \%$ & $12.3 \%$ & $13.2 \%$ \\
\hline 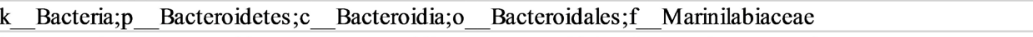 & $0.0 \%$ & $0.4 \%$ & $0.0 \%$ & $0.0 \%$ & $0.0 \%$ \\
\hline 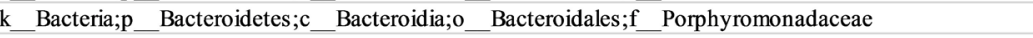 & $0.3 \%$ & $3.6 \%$ & $0.3 \%$ & $0.4 \%$ & $0.5 \%$ \\
\hline $\mathrm{k}$ _Bacteria; $\mathrm{p} \_$Bacteroidetes;c_Bacteroidia; $\mathrm{c}$ __Bacteroidales;f_Prevotellaceae & $3.7 \%$ & $3.8 \%$ & $2.6 \%$ & $3.7 \%$ & $2.9 \%$ \\
\hline k__Bacteria;p__Bacteroidetes;c__Bacteroidia;o__Bacteroidales;f_RF16 & $0.9 \%$ & $2.0 \%$ & $1.9 \%$ & $2.6 \%$ & $2.6 \%$ \\
\hline k_Bacteria;p_B_Bacteroidetes;c_Bacteroidia;o__Bacteroidales;f__ Rikenellaceae & $4.0 \%$ & $1.8 \%$ & $4.0 \%$ & $2.5 \%$ & $3.1 \%$ \\
\hline k_Bacteria;p__Bacteroidetes;c_Bacteroidia;o__Bacteroidales;f_S24-7 & $2.4 \%$ & $1.1 \%$ & $2.8 \%$ & $1.4 \%$ & $0.5 \%$ \\
\hline 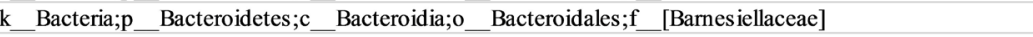 & $0.1 \%$ & $0.0 \%$ & $0.1 \%$ & $0.0 \%$ & $0.0 \%$ \\
\hline k_ Bacteria;p_Bacteroidetes;c_Bacteroidia;o_ Bacteroidales; $;$ [Odoribacteraceae] & $0.0 \%$ & $0.0 \%$ & $0.1 \%$ & $0.0 \%$ & $0.0 \%$ \\
\hline k__Bacteria;p_Bacteroidetes;c__Bacteroidia;o__Bacteroidales;f_[Paraprevotellaceae] & $5.4 \%$ & $1.2 \%$ & $4.1 \%$ & $4.8 \%$ & $4.9 \%$ \\
\hline $\mathrm{k} \_$_Bacteria;p_B_Bacteroidetes;c_Bacteroidia;o__Bacteroidales;f__ $\mathrm{p}-2534-18 \mathrm{~B} 5$ & $0.0 \%$ & $0.3 \%$ & $0.0 \%$ & $0.0 \%$ & $0.0 \%$ \\
\hline $\mathrm{k}$ __Bacteria;p_Chlorobi;c_oPB56;o_;f___ & $0.0 \%$ & $0.5 \%$ & $0.0 \%$ & $0.0 \%$ & $0.0 \%$ \\
\hline $\mathrm{k} \_$Bacteria;p_Cyanobacteria;c_4COd-2;0_ YS2;f & $0.4 \%$ & $0.3 \%$ & $0.2 \%$ & $0.3 \%$ & $0.5 \%$ \\
\hline 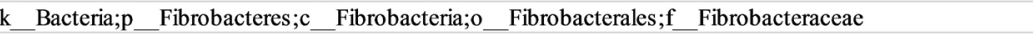 & $0.0 \%$ & $3.6 \%$ & $0.0 \%$ & $0.0 \%$ & $0.0 \%$ \\
\hline 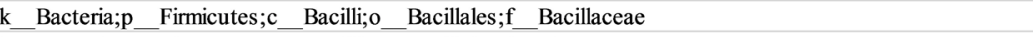 & $0.0 \%$ & $0.0 \%$ & $0.0 \%$ & $0.1 \%$ & $0.0 \%$ \\
\hline k__Bacteria;p_Firmicutes;c__Bacilli;o__Bacillales;f__Planococcaceae & $0.0 \%$ & $2.6 \%$ & $0.0 \%$ & $0.0 \%$ & $0.0 \%$ \\
\hline k__Bacteria;p__Firmicutes;c__Bacilli;o__Lactobacillales;f_Carnobacteriaceae & $0.0 \%$ & $0.0 \%$ & $0.0 \%$ & $0.0 \%$ & $0.1 \%$ \\
\hline k_Bacteria;p_Firmicutes;c_Clostridia;o_CClostridiales; Other & $2.4 \%$ & $1.1 \%$ & $2.3 \%$ & $1.6 \%$ & $1.2 \%$ \\
\hline k__Bacteria;p_Firmicutes;c_Clostridia;o__Clostridiales;f__ & $5.1 \%$ & $1.5 \%$ & $5.5 \%$ & $2.2 \%$ & $2.7 \%$ \\
\hline k__Bacteria;p_Firmicutes;c_Clostridia;o_Clostridiales;f_Christensenellaceae & $0.0 \%$ & $0.0 \%$ & $0.0 \%$ & $0.0 \%$ & $0.0 \%$ \\
\hline k__Bacteria;p__Firmicutes;c_Clostridia;o__Clostridiales;f_Clostridiaceae & $3.3 \%$ & $1.1 \%$ & $4.0 \%$ & $1.6 \%$ & $1.4 \%$ \\
\hline k__Bacteria;p_Firmicutes;c_CClostridia;o_CClostridiales;f_L_Lachnospiraceae & $8.8 \%$ & $6.2 \%$ & $10.6 \%$ & $9.6 \%$ & $7.1 \%$ \\
\hline k__Bacteria;p__Firmicutes;c_Clostridia;o__Clostridiales;f_Peptococcaceae & $0.0 \%$ & $0.0 \%$ & $0.1 \%$ & $0.1 \%$ & $0.1 \%$ \\
\hline k_Bacteria;p__Firmicutes;c_Clostridia;o_Clostridiales;f__Peptostreptococcaceae & $0.0 \%$ & $0.1 \%$ & $0.0 \%$ & $0.0 \%$ & $0.0 \%$ \\
\hline k_Bacteria;p_Firmicutes;c_Clostridia;o__Clostridiales;f_Ruminococcaceae & $23.3 \%$ & $16.2 \%$ & $26.1 \%$ & $22.0 \%$ & $21.7 \%$ \\
\hline k__Bacteria;p__Firmicutes;c_Clostridia;o_Clostridiales;f__Veillonellaceae & $3.5 \%$ & $2.5 \%$ & $3.2 \%$ & $3.7 \%$ & $3.7 \%$ \\
\hline k__Bacteria;p__Firmicutes;c_Clostridia;o__Clostridiales;f_[Mogibacteriaceae] & $0.5 \%$ & $0.6 \%$ & $0.6 \%$ & $0.6 \%$ & $0.3 \%$ \\
\hline 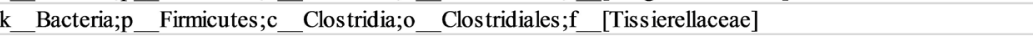 & $0.0 \%$ & $0.1 \%$ & $0.0 \%$ & $0.0 \%$ & $0.0 \%$ \\
\hline k__Bacteria;p__Firmicutes;c_Erysipelotrichi;o_Erysipelotrichales;f_Erys ipelotrichaceae & $0.7 \%$ & $1.1 \%$ & $0.7 \%$ & $0.9 \%$ & $0.7 \%$ \\
\hline k_Bacteria;p_Fusobacteria;c_Fusobacteriia;o__Fusobacteriales;f_Fusobacteriaceae & $0.0 \%$ & $0.1 \%$ & $0.0 \%$ & $0.0 \%$ & $0.0 \%$ \\
\hline 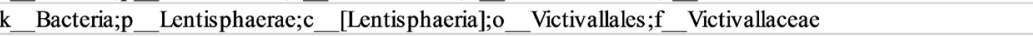 & $0.0 \%$ & $0.1 \%$ & $0.0 \%$ & $0.0 \%$ & $0.0 \%$ \\
\hline k__Bacteria;p__Proteobacteria;c_Alphaproteobacteria;o__RF32;f & $0.1 \%$ & $0.0 \%$ & $0.1 \%$ & $0.1 \%$ & $0.1 \%$ \\
\hline 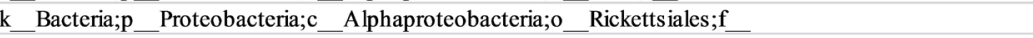 & $0.1 \%$ & $0.1 \%$ & $0.0 \%$ & $0.1 \%$ & $0.0 \%$ \\
\hline $\mathrm{k} \_$Bacteria; $\mathrm{p} \_$Proteobacteria;c__Betaproteobacteria;o__Burkholderiales;f__ Alcaligenaceae & $0.3 \%$ & $0.9 \%$ & $0.2 \%$ & $1.1 \%$ & $0.5 \%$ \\
\hline k_Bacteria;p__Proteobacteria;c_Betaproteobacteria;o__Burkholderiales;f_CComamonadaceae & $0.0 \%$ & $4.3 \%$ & $0.0 \%$ & $0.0 \%$ & $0.0 \%$ \\
\hline k__Bacteria;p__Proteobacteria;c__Betaproteobacteria;o__Rhodocyclales;f__Rhodocyclaceae & $0.0 \%$ & $0.1 \%$ & $0.0 \%$ & $0.0 \%$ & $0.0 \%$ \\
\hline k__ Bacteria;p_Proteobacteria;c___Deltaproteobacteria;o__Desulfovibrionales;f__Desulfovibrionaceae & $0.0 \%$ & $0.4 \%$ & $0.1 \%$ & $0.4 \%$ & $0.9 \%$ \\
\hline k_ Bacteria;p_Proteobacteria;c_Epsilonproteobacteria;o Campylobacterales;f Campylobacteraceae & $0.0 \%$ & $7.2 \%$ & $0.0 \%$ & $0.2 \%$ & $0.3 \%$ \\
\hline k_ Bacteria;p_Proteobacteria;c__ammaproteobacteria;o_Aeromonadales;f__ Succinivibrionaceae & $6.6 \%$ & $8.4 \%$ & $6.1 \%$ & $11.4 \%$ & $16.4 \%$ \\
\hline k__ Bacteria;p__Proteobacteria;c__ammaproteobacteria;o__Enterobacteriales;f__Enterobacteriaceae & $0.1 \%$ & $0.1 \%$ & $0.0 \%$ & $0.0 \%$ & $0.0 \%$ \\
\hline k__Bacteria;p_Proteobacteria;c__Gammaproteobacteria;o_Pseudomonadales;f__ Moraxellaceae & $0.0 \%$ & $1.0 \%$ & $0.1 \%$ & $0.0 \%$ & $0.0 \%$ \\
\hline k_Bacteria;p_Proteobacteria;c_Gammaproteobacteria;o_Pseudomonadales;f__Pseudomonadaceae & $0.0 \%$ & $0.1 \%$ & $0.0 \%$ & $0.0 \%$ & $0.0 \%$ \\
\hline k_Bacteria;p_Proteobacteria;c_Cammaproteobacteria;o_Xanthomonadales;f_Xanthomonadaceae & $0.0 \%$ & $0.4 \%$ & $0.0 \%$ & $0.0 \%$ & $0.0 \%$ \\
\hline k_Bacteria;p_Spirochaetes;c_Spirochaetes;o__Spirochaetales;f__Spirochaetaceae & $2.7 \%$ & $2.6 \%$ & $4.0 \%$ & $5.0 \%$ & $4.8 \%$ \\
\hline k_Bacteria; & $0.6 \%$ & $0.3 \%$ & $0.5 \%$ & $0.2 \%$ & $0.0 \%$ \\
\hline k_Bacteria;p_Tenericutes;c_Mollicutes;o__Acholeplasmatales;f_Acholeplasmataceae & $0.0 \%$ & $0.5 \%$ & $0.0 \%$ & $0.0 \%$ & $0.0 \%$ \\
\hline k_Bacteria;p_Tenericutes;c_Mollicutes;o_Anaeroplasmatales;f_Anaeroplasmataceae & $0.2 \%$ & $0.4 \%$ & $0.1 \%$ & $0.3 \%$ & $0.5 \%$ \\
\hline k__Bacteria;p_Tenericutes;c_Mollicutes;o_RF39;f_ & $0.6 \%$ & $0.1 \%$ & $0.6 \%$ & $0.2 \%$ & $0.3 \%$ \\
\hline k_Bacteria;p_Tenericutes;c_RF3;o__ML615J-28;f_ & $0.0 \%$ & $0.1 \%$ & $0.0 \%$ & $0.1 \%$ & $0.2 \%$ \\
\hline k_Bacteria;p_Verrucomicrobia;c Opitutae;o_[Cerasicoccales];f_[Cerasicoccaceae] & $0.0 \%$ & $0.0 \%$ & $0.0 \%$ & $0.0 \%$ & $0.1 \%$ \\
\hline k_Bacteria;p_Verrucomicrobia;c_Verruco-5;o_WCHB1-41;f RFP12 & $0.0 \%$ & $0.2 \%$ & $0.0 \%$ & $0.0 \%$ & $0.0 \%$ \\
\hline $\mathrm{k} \_$_Bacteria; $\mathrm{p} \_$Verrucomicrobia;c_Verrucomicrobiae;o_Verrucomicrobiales;f_Verrucomicrobiaceae & $1.0 \%$ & $0.6 \%$ & $0.5 \%$ & $0.6 \%$ & $0.7 \%$ \\
\hline
\end{tabular}

Figure S1. Percentage of prokaryotic taxa changes at the family level. Prokaryotic families detected in the manure were assessed for their percent composition of the whole population (detectable range $\geq 0.1 \%$ ). The percentages were compared to fresh manure and increases were denoted in green, decreases in red, and no change in yellow. 